\title{
L'intentionnalité des émotions dans l'analyse du discours radiophonique. Étude de cas d'une émission informative colombienne
}

\author{
Natalia Marcela Osorio Ruiz ${ }^{1, *}$ \\ ${ }^{1}$ Laboratoire LHUMAIN, Université Paul Valéry Montpellier 3, 34000 Montpellier, France
}

\begin{abstract}
Résumé. Cet article vise à observer les manifestations émotionnelles qui émergent dans le discours radiophonique informatif d'une station colombienne, Caracol Radio et, plus particulièrement, de son émission matinale «6 AM Hoy por hoy». Dans une démarche ethnographique, cette recherche s'appuie sur un corpus où les enregistrements vidéo réalisés en studio donnent accès à des détails spécifiques du professionnalisme journalistique. Avec une approche d'étude qualitative et longitudinale, cette contribution vise à analyser le traitement des émotions dans deux extraits. Cette étude a été réalisée sous le prisme du " parcours émotionnel », tel que défini par Plantin, Traverso et Vosghanian [17], et la différence entre une émotion intentionnellement manifestée et une émotion intentionnellement cachée. Dans l'analyse effectuée, la multimodalité est prise en compte. En effet, l'aspect corporel des acteurs médiatiques ainsi que les changements phono-prosodiques sont considérés comme significatifs, car la visée pathémique est construite par la situation dans son ensemble.
\end{abstract}

\begin{abstract}
This article aims to observe the emotional manifestations emerging in the informative radio's speech of a Colombian radio station, Caracol Radio and, more particularly, its morning broadcast «6 AM Hoy por hoy». Grounding on an ethnographic approach, this research is based on a corpus where video recordings made in studio give access to specific details of journalistic professionalism. Based on a qualitative and longitudinal study, this contribution aims to analyse the treatment of emotions in two extracts. This study was made under the prism of the "emotional journey", as defined in Plantin, Traverso and Vosghanian [17], and the difference between an emotion intentionally manifested and an intentionally hidden emotion. The analysis takes multimodality account. Indeed, embodied aspects as well as phonetic-prosodic changes are considered meaningful, because the pathemic aim is built by the situation in its entirety.
\end{abstract}

므amaosru27@gmail.com 


\section{Introduction}

Le discours médiatique poursuit différents buts selon le type de média, de format ou d'intérêt éditorial. Néanmoins, il est important de retenir que tout type de discours intègre un double ancrage : rationnel et émotionnel. En effet, même en cherchant à n'être que strictement rationnel et logique, le discours inclut l'aspect émotionnel puisque «représentation rationnelle et émotion sont portées par les mêmes mots, les mêmes constructions, les mêmes arguments ; elles portent aux mêmes intentions de discours » [1, p. 2]. Cette indissolubilité est essentielle au discours humain et a été repérée assez rapidement, puisque, dès la rhétorique d'Aristote, la projection des émotions chez l'auditoire, le pathos, a été considérée comme un des piliers discursifs servant à la persuasion. Les médias ont comme but premier d'être crus et de susciter une adhésion de leurs publics, ce qui leur permettra de les fidéliser. La présente contribution cherche ainsi à observer le fonctionnement de cette manifestation émotionnelle dans le discours radiophonique informatif d'une chaîne de radio colombienne. Pour cela, il sera question dans un premier temps d'aborder certaines caractéristiques de l'intentionnalité au sein du discours radiophonique. Dans un deuxième temps, seront exposés le contexte de cette recherche effectuée au sein d'une émission en particulier et la démarche ethnographique qui la sous-tend. Seront enfin présentés et étudiés deux extraits qui attestent d'un traitement intentionnellement différencié des émotions.

\section{L'intentionnalité dans le discours radiophonique}

«Selon une tradition rhétorique, la parole radiophonique renvoie à un pathos, au sens de l'influence affective que la voix exerce sur les publics. Elle n'en exhibe pas moins avant tout un ethos » [2, p. 99]. Cette citation nous rappelle que, dans un média comme la radio, qui est de nature exclusivement sonore, la complémentarité entre l'ethos et le pathos s'opère en grande partie sur les aspects prosodiques et phonétiques, mais aussi sur la parole elle-même (le logos qui complète le triangle rhétorique aristotélicien). Le discours radiophonique se structure, se pré-construit et se construit en continuum selon des fins visées. En effet, d'après Quéré [3], la manière dont le discours médiatique est reçu se façonne dès la production. Ainsi, la visée pathémique, consistant à susciter des émotions, est considérée selon Charaudeau comme un des effets possibles du discours, possible car « une certaine mise en discours [peut susciter une certaine réaction] auprès d'un certain public, dans une certaine circonstance » [4]. C'est pourquoi une bonne connaissance du public détermine la réussite ou l'échec de la construction de l'émotion. Cette recherche d'émotion à travers le discours s'appuie ainsi sur le lien qui se tisse entre la raison et les émotions. Ce lien accorde la propriété d'intentionnalité aux émotions. Intentionnalité non au sens de la tradition pragmatique, par rapport à la théorie des actes de langage d'Austin et Searle, mais au sens de Charaudeau de la visée représentationnelle ou pathémique en tant que stratégie de capture d'auditoire (au sein de la tradition rhétorique latine). En effet, « c'est parce que les émotions se manifestent dans un sujet "à propos" de quelque chose qu'il se figure, qu'elles peuvent être dites intentionnelles » [5]. Raison pour laquelle elles font partie du fonctionnement rhétorique des médias.

Nous visons à observer l'intentionnalité de susciter ou pas de l'émotion chez l'auditoire dans le discours radiophonique, à travers deux études de cas récoltées par nos soins. Il est à noter que la plupart des études sur le discours radiophonique se centrent sur le produit final, l'émission telle qu'elle est reçue par les auditeurs ([6], [7], [8]). Mais il existe très peu de travaux avec des immersions sur le terrain ([9], [10], [11]). C'est pourquoi il nous semble pertinent d'aborder brièvement notre ancrage méthodologique. 


\section{Au sein de « Caracol Radio »}

Grâce à une démarche ethnographique réalisée au sein d'une chaîne de radio informative colombienne, cette étude dispose d'un corpus multimodal qui permet d'étudier la construction du discours radiophonique. La chaîne de radio s'appelle « CARACOL RADIO», nom résultant d'une apocope, avec un sens autonome, de CAdena RAdial COLombiana. Elle est née en 1948 et est une chaîne de radio à diffusion nationale. Elle est une entreprise privée qui appartient au groupe espagnol PRISA depuis 2004. Le programme « 6 AM Hoy por hoy », que l'on pourrait traduire par « $6 \mathrm{~h}$ du matin, au jour le jour », est notre principal objet d'étude. C'est l'émission informative de la matinale qui, selon les études d'audience, est la plus écoutée de la radio colombienne. Depuis 1979, ce programme est diffusé du lundi au vendredi entre $4 \mathrm{~h}$ et $11 \mathrm{~h}$ pour une écoute massive de $6 \mathrm{~h}$ à $9 \mathrm{~h}$, et un passage en format radio visuelle à la télévision de $6 \mathrm{~h}$ à $8 \mathrm{~h}$. Cette émission est cadencée comme suit : de $4 \mathrm{~h}$ à $6 \mathrm{~h}$ il y a des bulletins informatifs sur les différentes régions du pays, avec des reportages faits par les journalistes de terrain. Entre $6 \mathrm{~h}$ et $9 \mathrm{~h}$, il y a une focalisation majeure sur les informations du jour et les affaires politico-économiques. Ensuite, une séquence de deux heures ouvre l'antenne à des sujets d'intérêt général, des conseils et des expériences personnelles. Cette partie du programme se fait sous forme de magazines et chroniques orientés vers le social puisqu'elle offre la possibilité aux auditeurs de prendre connaissance de certains récits de vies ordinaires, mais exemplaires, en insistant sur les cas de résilience par exemple. En outre, des conseils sont proposés de la part d'experts dans différents domaines, sous forme de conseils à l'appui, et enfin cette section donne la possibilité de faire des dénonciations sociales en relayant des lanceurs d'alerte.

L'étude de cette émission a été l'objet d'un travail de terrain en immersion de trois mois au sein de l'équipe de rédaction, en mars et en juillet 2016 et en août 2017. Ce corpus se compose alors de journaux de terrain, de vidéos en studio, en production et en conférences de rédaction, de podcasts du programme, de tweets, d'entretiens effectuées avec les journalistes avec des enregistrements sonores et parfois vidéo, de scripts du programme et des captures d'écran qui révèlent la communication entre le producteur et les journalistes. L'objectif de ce travail de terrain ne se limitait pas exclusivement à la récolte passive des données. En effet, c'est l'observation participante qui a donné l'opportunité de connaître le déroulement des actions, et aussi, de réaliser des tâches des professionnels des médias. C'est par ce biais que l'approche ethnométhodologique de Garfinkel [12] assumée s'est vue renforcée en travaillant avec eux de l'intérieur et en essayant de saisir la signification qu'ils attribuent aux actions. Cette immersion a permis de comprendre le pourquoi des procédures et donc la logique du fonctionnement interne.

D'ailleurs, l'instrumentation employée dans la récolte des données a été justifiée par l'enrichissement de l'apport désormais classique des instruments et de la technologie pour l'investigation de recherche à l'ethnographie. En effet, d'après Duranti [13], ces outils permettent d'effectuer des descriptions plus profondes en motivant une analyse complexe qui prend en compte tous les éléments, notamment ceux de nature multimodale. Les enregistrements des actions quotidiennes de ces professionnels dévoilent les gestes de métier et relèvent de la naturalité des comportements. Ainsi, l'ethnographie liée à l'ethnométhodologie constituent une voie d'accès aux pratiques des acteurs. Ces dernières sont des activités qui leur sont quotidiennes et donc naturelles, mais qui ont la condition d'être des faits « detectable [s], countable, recordable, reportable, tell-a-story-aboutable, analyzable-in short, accountable » [12, p. 33]. Ainsi, nous pouvons saisir les points de vue des agents, dans notre cas, des journalistes, afin de comprendre le sens qu'ils attribuent à leurs actions ${ }^{\dagger}$. Ces dernières obéissent en effet à de " socially organized common practices »

\footnotetext{
${ }^{\dagger}$ Un autre exemple de cette démarche se trouve dans [11].
} 
[12, p. 33]. De cette manière, nous visons à étudier la construction du discours radiophonique comme une action en contexte, une action in situ. Finalement, le cadre croisé de l'apport ethnométhodologique et de l'analyse du discours permet de questionner les fonctionnements participatifs de la parole journalistique, en la reliant aux enjeux de sa portée sur les publics. En effet, l'analyse du discours « associe intimement langue (plus largement les ressources sémiotiques disponibles dans une société), activité communicationnelle et connaissance (les divers types de savoirs, individuels ou collectifs, mobilisés dans la construction du sens des énoncés) » [14, p. 25]. Prenant ainsi l'ensemble des dimensions qui traversent une production discursive, dans notre cas de nature médiatique, depuis un point de vue micro, en tant que production langagière, vers un point de vue macro, en tant qu'activité sociale.

\section{3 Étude de cas}

L'approche de cette étude est qualitative et longitudinale à des fins d'illustration. En effet, elle vise à illustrer à travers deux exemples le fonctionnement de la manifestation émotionnelle dans le discours radiophonique. Pour cet article, une focalisation particulière est proposée sur l'intentionnalité mise en évidence dans le traitement de l'émotion pour deux cas précis. Pour le premier, il est question de l'élaboration du pathos, compris comme le souhait de créer chez l'auditoire une certaine émotion. Pour le second, à l'inverse, le souhait est de ne pas mettre en évidence l'émotion ressentie, en obéissant aux paramètres du contrat de professionnalité implicitement (ou parfois explicitement) établi. Dans ces deux cas, les vidéos enregistrées en studio permettent d'avoir accès à la mimogestualité qui peut dévoiler l'intentionnalité de susciter ou non de l'émotion chez l'auditoire.

\subsection{L'émotion manifestée}

Le premier cas correspond à l'entretien réalisé le 15 août 2017 avec un jeune garçon dont la sœur a une paralysie cérébrale suite à une injection mal appliquée. Comme l'expliquera la journaliste, l'invité a décidé de parcourir avec elle l'Amérique du Sud afin de chercher une solution pour cette pathologie et de récolter de l'argent pour une chirurgie pouvant améliorer sa qualité de vie. L'intérêt de l'étude a consisté à observer comment le média radiophonique vise à susciter de l'émotion chez l'auditeur. C'est pourquoi il y a une focalisation sur le parcours émotionnel co-construit entre les journalistes, en tant qu'acteurs médiatiques. Il est à noter que même si, pendant l'analyse, les énoncés des journalistes forment le point central d'étude, en aucun cas les réponses données par l'invité ne sont exclues puisqu'elles font partie d'une séquence conversationnelle qui évolue au fur et à mesure que l'interaction avance.

La séquence d'ouverture correspond à l'introduction de l'histoire *:

\section{(1) Introduction de l'entretien}

* délimitation des actions corporelles de DAR

+ délimitation des actions corporelles de PAT

1. DAR ahora son las diez de la mañana nueve minutos maintenant c'est dix heures du matin et neuf minutes $\backslash$

\section{DAR esta his:toria es verdaderamente: asombrosa y}

\footnotetext{
‡ Les transcriptions sont réalisées suivant la convention ICOR 2013 faite par le Groupe ICOR UMR 5191 ICAR (CNRS - Lyon 2 - ENS de Lyon). Le verbal en espagnol est en gras, la traduction en deuxième ligne et les notations multimodales, quand présentes, en troisième ligne en italique. Ces dernières ont été réalisées en suivant les conventions de Mondada, L. (2018) [15].
} 


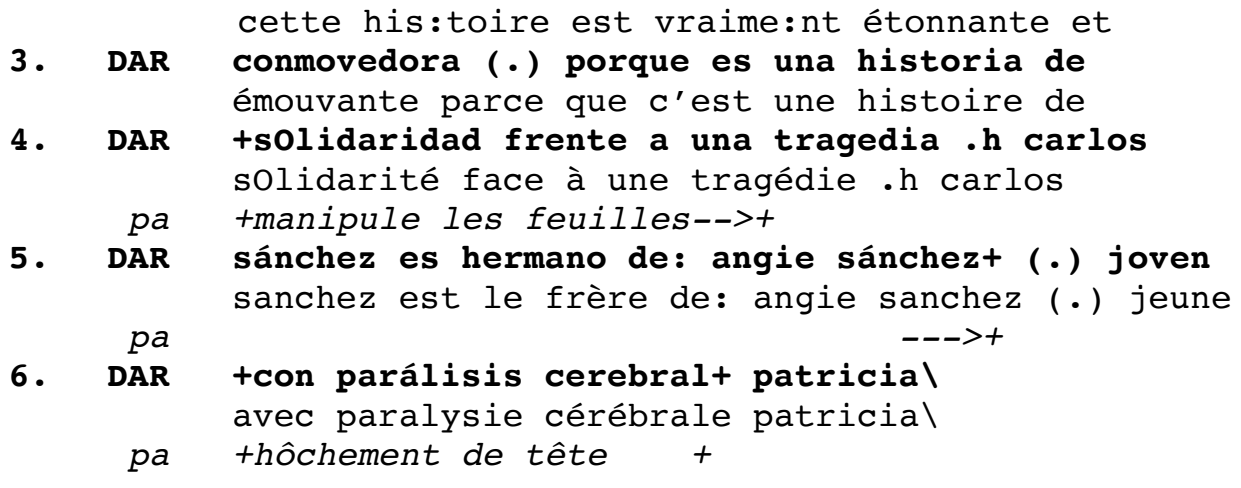

Cette introduction prend appui sur des adjectifs qualificatifs mélioratifs tels que « étonnante et émouvante », l'adverbe d'affirmation « vraiment » et deux groupes nominaux mis en contraste « une histoire de solidarité » et « une tragédie ». De cette manière, il y a un façonnement préalable à la présentation du récit quant à la manière de le recevoir, faisant allusion au façonnement de la réception dès la production étudiée par Quéré [3] ou du « recipient design » [16] dans le cadre de l'analyse de conversation. Cette introduction inclut une tournure argumentative autour de la qualification effectuée. DAR en premier énoncé attribue lesdits adjectifs à l'histoire et, par la suite, emploie la conjonction « parce que » afin d'expliquer la raison de son propos. Ainsi, il réalise une auto-validation de son énoncé, limitant la place laissée au doute.

Après cette introduction par le directeur de l'émission, PAT prend le tour de parole et présente à son tour la situation.

(2) Prise de tour de parole par PAT
7. PAT
sil eh: darío y ellos: pues decidieron
8. PAT
ouil euh: dario et ils: bon ils ont décidé de
9. PAT
recorrer suramérica .h (.) buscando una
parcourir l'amérique du sud (.) en cherchant une
10. PAT
solución para esa parálisis cerebral que
solution pour cette paralysie cérébrale dont
sufre esta niña o que padece o con la que vive souffre cette fille dont elle est atteinte ou avec laquelle elle vit
11. PAT angie eh después de una inyección maL Aplicada angie eh après une injection mAL Appliquée

Dans son tour de parole, la journaliste énonce le nom de la maladie qui touche la personne concernée et elle le fait en recourant à trois syntagmes verbaux « souffrir / être atteinte / vivre avec » qui tracent une séquence consécutive. Ensuite, elle énonce la cause avec une montée d'intensité sur les syllabes des dernières unités du tour de parole : «mAL Appliquée ». Cela engendre une réaction de la part du directeur du programme avec une emphase prosodique à nouveau qui porte sur la mauvaise procédure qui a causé cette tragédie comme il l'a qualifiée précédemment.

\section{(3) Réaction de DAR}

12. DAR *imagínese usted lo que puede generar una mA:la imaginez-vous ce que peut générer une mAU:vaise *en regardant $P A T--->$ 


\section{DAR aplicación de una* inyección\ \\ application d'une injection \}

Cette introduction pose déjà le cadre émotionnel de l'entretien. En effet, la présentation de l'histoire est déroulée à travers l'élaboration d'un parcours émotionnel, lui-même réalisé principalement entre la journaliste qui approfondit l'histoire, Patricia (PAT) et le directeur du programme, Dario (DAR). Précisons que la notion de "parcours émotionnel » est comprise au sens de sa conception en tant qu'unité d'analyse séquentielle. Plus précisément, cela fait référence à la définition de Plantin, Traverso et Vosghanian : « Il s'agit [donc] d'une unité co-construite par les participants, et non d'une unité produite par un seul locuteur (un tour de parole) et moins encore d'une unité isolée de son contexte (un mot ou un énoncé) » [17]. Ainsi, les deux journalistes, de par leurs échanges, visent à susciter de l'empathie chez l'auditeur face au vécu des frères protagonistes du récit. Cette procédure continue tout au long de l'entretien, où les deux journalistes (principalement mais pas exclusivement) demandent des détails, probablement pour permettre à l'auditoire une meilleure compréhension de la situation dans laquelle se retrouve cette jeune fille et de l'impact que la maladie a sur sa vie.

\section{(4) Demande des détails ${ }^{\S}$}

\section{DAR óigame carlos/ cómo se le manifiesta a ella écoutez-moi carlos/ comment se manifeste chez elle \\ 98. DAR esa: parálisis cerebral espásttica (.) cutáles cette: paralysie cérébrale spAstique(.) quelles

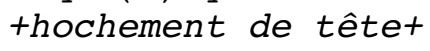

Cette procédure se justifie par le besoin de présenter le contexte de la situation pour aboutir à son objectif : créer de l'émotion chez l'auditoire. Comme le précisent Plantin, Traverso et Vosghanian "c'est [effectivement] le sens contextuel, et pas l'événement référence brut qui est alors responsable de l'émotion » [17]. En effet, c'est la description environnante qui permet d'orienter le type d'émotion à susciter chez l'auditoire, surtout quand ce public, sous le rôle de tiers symbolisant [18], ne peut pas interagir directement. De plus, nous pouvons observer que cette demande de détails est validée par l'autre journaliste, manifestant une fois de plus la co-construction de l'entretien par les acteurs médiatiques.

Ce parcours est réalisé sur le plan discursif mais aussi sur le plan gestuel. Certains mouvements s'accomplissent avec un but illustratif des mots énoncés, comme dans cette courte séquence.

(5) Emploi illustratif des gestes

\section{DAR clarol pero entonces cómo se *agarra usted bien sûr $\backslash$ mais alors comment vous vous agrippez *représentation}

\footnotetext{
${ }^{\S}$ Les passages non analysés en détail dans cet article ne sont pas cités, mais la numérotation correspond à l'extrait original.
} 
112. DAR

para +resistir un+ viaje en moto tan largo//*

pour résister un voyage en moto si long//

pa

avec les mains du geste de \# s'accrocher*

im

thochement de tête+

\section{J02 >si $<<$}

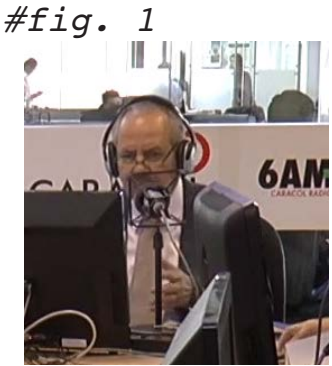

Fig. 1

\section{DAR como el que está haciendo en este momento comme celui que vous êtes en train de faire en ce moment}

Au moment où le directeur pose une question, il reproduit avec ses mains le geste de s'agripper pour illustrer ses propos (Fig. 1). Cela donne lieu à une interaction gestuelle avec PAT qui acquiesce pendant sa question, ainsi qu'à une courte interaction discursive avec JO2 qui énonce un « oui » rapide à la fin de la question et avant une phrase explicative de DAR. Les gestes constituent un moyen de communication très récurrent pour les journalistes radiophoniques, qui ne pouvant pas user de la parole à tout moment, se co-valident et coconstruisent l'interaction de manière multimodale (regards, gestes, expressions faciales) au sein du studio.

Relevant du même plan, des gestes qui exposent les réactions des journalistes face au discours entendu peuvent être observés. Au cours de l'entretien se trouve ainsi l'exemple suivant.

(6) Réaction gestuelle de PAT

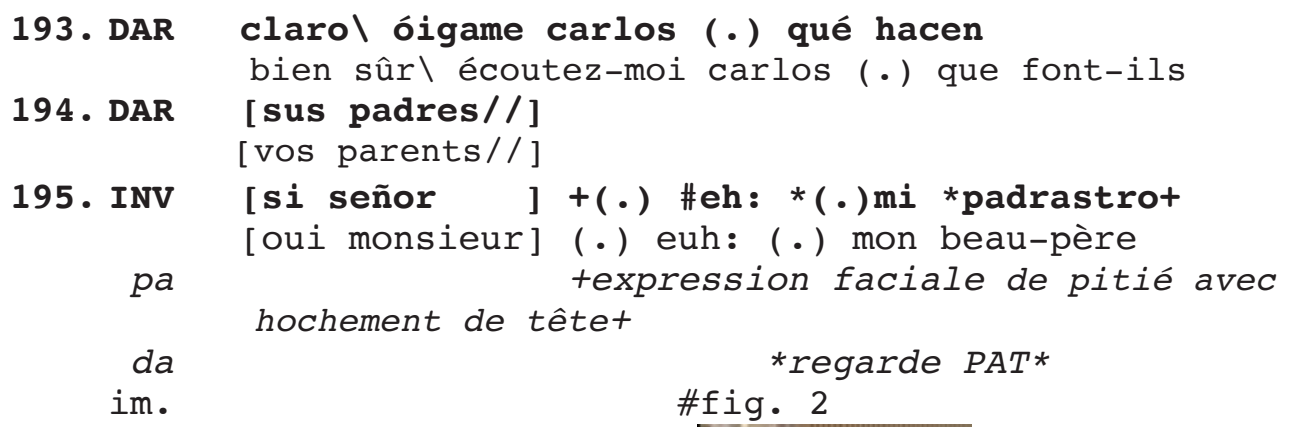

195. INV [si señor ] +(.) \#eh: *(.) mi *padrastrot [oui monsieur] (.) euh: (.) mon beau-père

da

im.

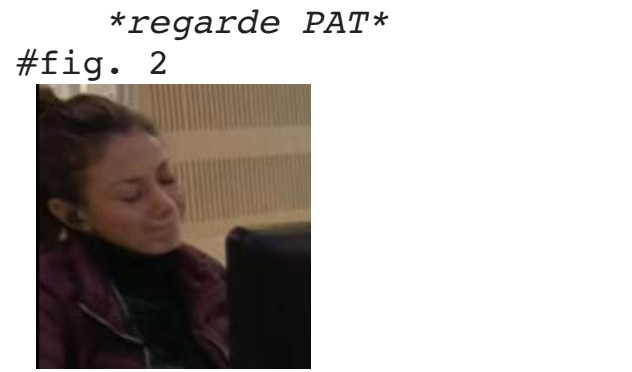




\section{Fig 2}

Lorsque DAR demande la profession des parents de l'invité, PAT réalise une expression faciale qui dénote une certaine émotion, laquelle pourrait être associée à la pitié. Il convient de préciser qu'elle connaît par avance la réponse à la question de DAR car c'est elle qui a cherché cette histoire et contacté la personne pour l'interview. Autrement dit, elle connaît la situation de précarité de la famille et c'est cela qui peut justifier sa réaction corporelle à la question.

Cette dimension corporelle, même si elle n'est pas visible pour les auditeurs, est accompagnée parfois par des marqueurs perceptibles à la voix. L'élaboration de la situation dans sa totalité permet de construire la visée pathémique. En effet, les changements phonético-prosodiques, les intonations montantes dans certains mots (mAL Aplicada), ou les allongements vocaliques (bue:na energía) focalisent l'attention de l'auditoire. Ces ressources deviennent significatives et porteuses de sens à la radio par l'emballage prosodique que constitue la voix en tant qu'actualisatrice de la parole, comme le précise Burger et Auchlin [8].

De plus, sur le plan discursif l'entretien est accompagné d'énoncés subjectifs produits par les journalistes, de diminutifs et de qualifications. Voici l'exemple d'une qualification explicite co-construite se référant au frère.

(7) Qualification co-construite

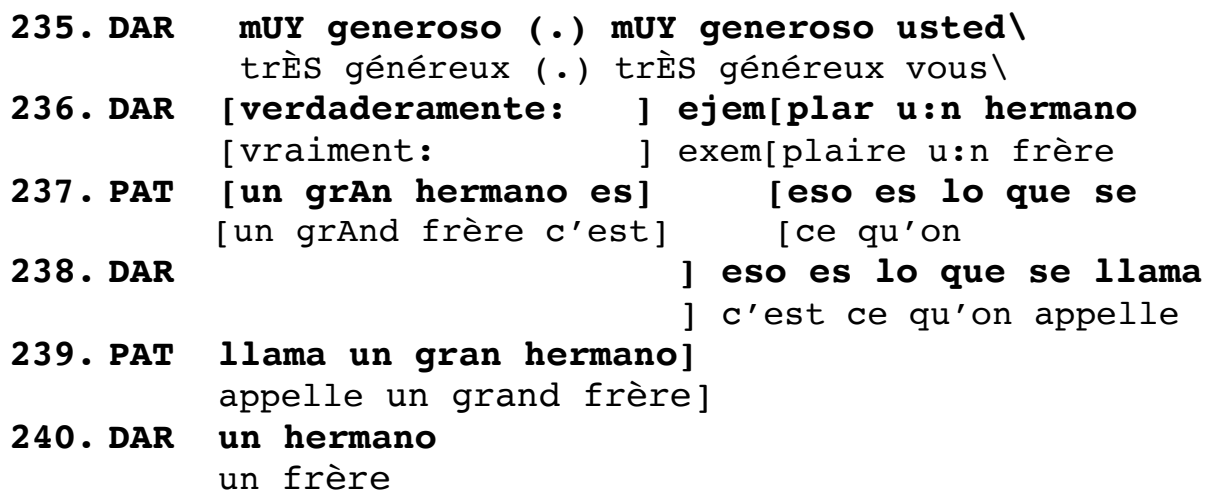

Ainsi, cette qualification co-construite expose un accord de la part des journalistes, mais aussi, à travers l'emploi des énoncés impersonnels, manifeste une inclusion de l'auditoire. En effet, l'emploi d'un effacement énonciatif permet de placer la situation comme «allant de soi ", ce qui constitue un appel au partage social des émotions. De cette manière, une adhésion du public est suscitée quant aux propos axiologiques et par ricochet à l'empathie. Ce souhait est manifesté directement en clôture de l'entretien.

(8) Clôture d'entretien
244. PAT pues carlos muchísimas gracias y: (.) ojalÁ bon carlos merci beaucoup et: (.) j'espeRE
245. PAT gente de buen corazón nos esté escuchando y que que des gens avec un bon cœur sont en train de nous écouter et

246. PAT quiera: colaborarles que quiera ayudarles o por qu'ils voudront collaborer avec vous qu'ils voudront vous aider ou

247. PAT [lo menos que le quieran env]iar una bue:na 


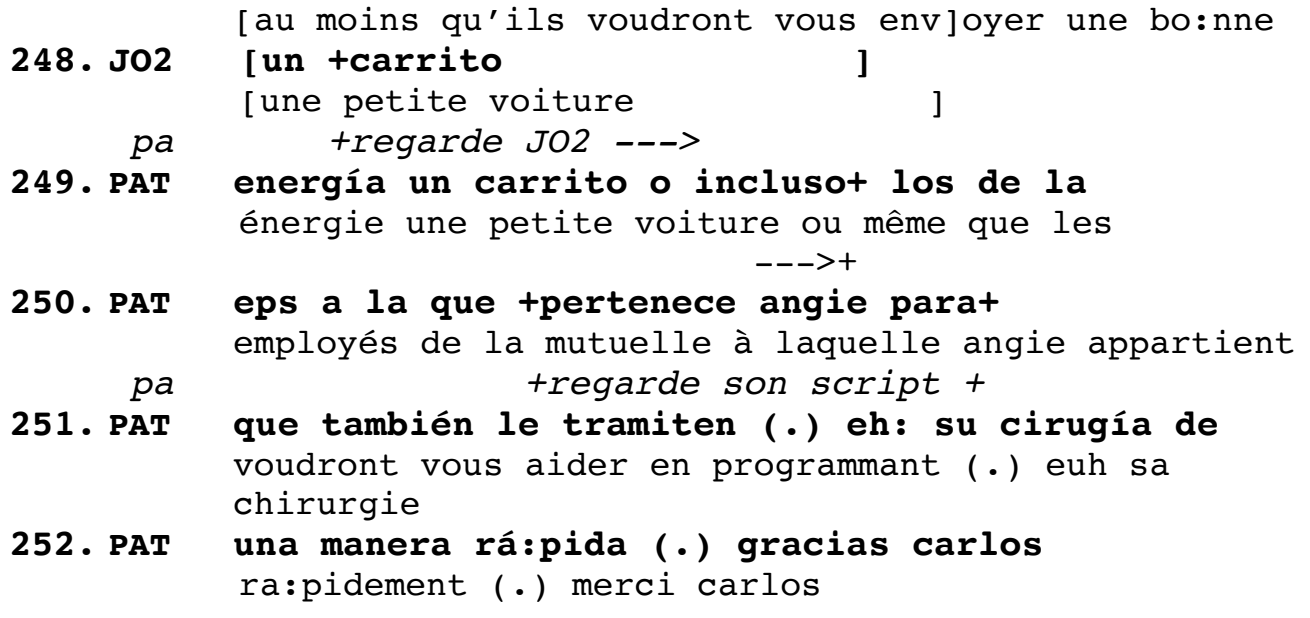

Comme nous pouvons l'observer, la clôture passe par une invitation à aider le frère et la sœur. Cette aide est sollicitée par le biais d'un appel à l'empathie des auditeurs, qui de fait sont catégorisés comme «des gens avec un bon cœur». Cette catégorisation imprime une certaine contrainte sur les auditeurs puisque d'une manière sous-entendue pour pouvoir faire partie de ce groupe il faut aider ou avoir de l'empathie pour la fratrie. De même, il y a un appel au personnel de l'assurance maladie de la patiente mais sans préciser son nom.

\subsection{L'émotion dissimulée}

Avant de présenter la séquence il est nécessaire d'expliquer la distribution de l'espace de travail. Le studio de diffusion est séparé du studio de production par une vitre. L'opérateur technique se situe en face de la table en forme d'arc, ce qui lui permet d'avoir un contact visuel avec tous les journalistes et un contact verbal médié par les dispositifs technologiques (casques et microphones) (Fig. 3).

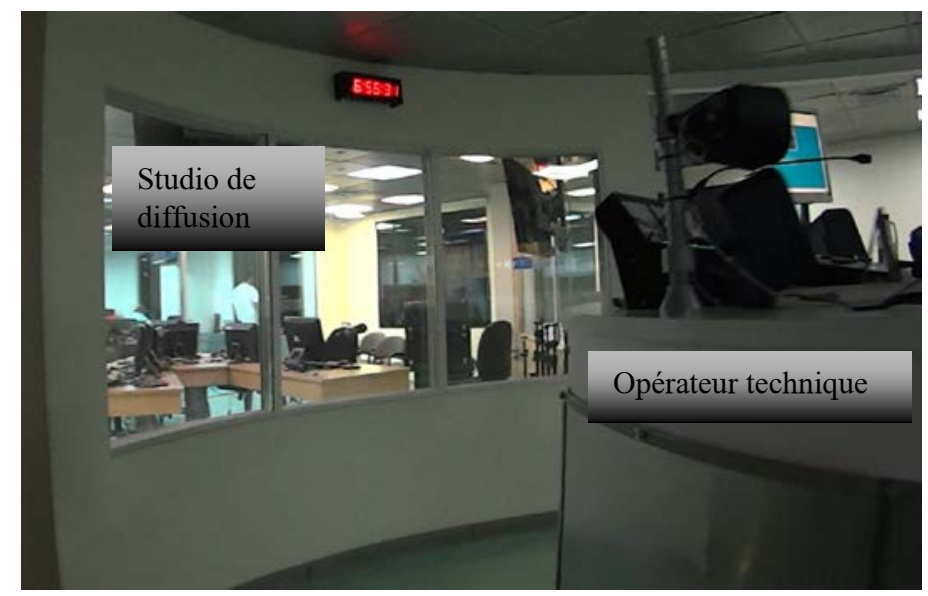

Fig. 3. Studio de diffusion et studio de production

Grâce à cette distribution spatiale, les participants peuvent échanger continuellement, ce qui est indispensable pour le bon déroulement de l'émission. En effet, "la visibilité symétrique est un aspect crucial de l'espace de travail qui garantit la cohérence interactionnelle par la coprésence et par le partage d'une même conscience de cet environnement d'artefacts et d'interactions » [10, p. 4]. La visibilité de l'opérateur technique 
est spécialement importante, car c'est lui qui fait retentir les sons à l'antenne et qui contrôle l'ouverture et la fermeture des microphones.

En revenant à la situation précise, ce second cas correspond à une séquence où il est question de dissimuler l'émotion ressentie. En effet, dans un extrait du 30 août 2017, l'opérateur technique fait une faute dans le déroulement de l'émission puisqu'il fait retentir à l'antenne le jingle d'introduction à une séquence précise, à savoir « les secrets de Darcy Quin », alors que ce n'est pas encore le moment**.

\section{(9) Introduction de section}

* délimitation des actions corporelles de DAR

$\S$ délimitation des actions corporelles de DCY

1. son da fig

2. DAR no*: $(0.2) * \$(1.2)$

$<((j$ ingle $))$ los secretos *de \#darcy quinn*> $<((j i n g l e))$ les sécrets de darcy quinn> *ouvre les bras* \#fig. 4

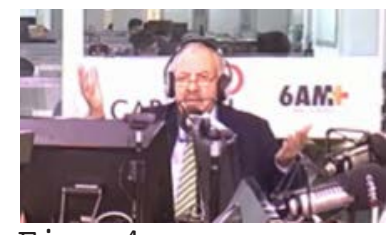

Fig. 4

*bouge les bras, tape la table de sa main droite tout en inclinant sa tête*

dc Sregard étonné vers l'opérateur--->

3. son ((jingle \#\$ sonore))

fig

4. DAR

Fig. 5

\#fig. 5

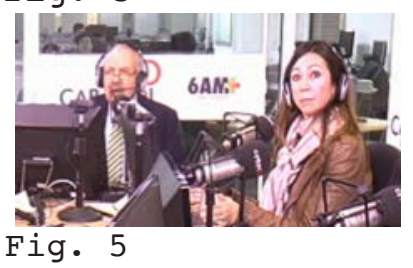

es e:l primer se*creto de darcy*

c'est le: premier secret de darcy

*faisant des négations avec la

5. DAR *qUINn\# en: cara*col

tête*

quINn à: caracol\

* levant son bras droit*

\footnotetext{
** Pour faciliter la compréhension les énoncés entendus à l'antenne sont en noir gras, et ceux entendus hors antenne en gris gras. Les transcriptions des silences ont été faites d'après les indications de Mondada, 2019 [19].
} 
fig

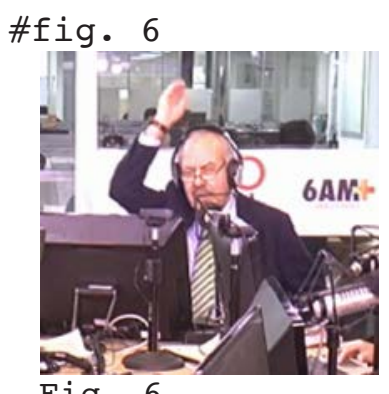

Fig. 6

Grâce à la vidéo en studio, nous pouvons observer la surprise de toute l'équipe et l'énervement du directeur, perceptible par ses gestes de mécontentement même pendant le passage à l'antenne. Malgré cette émotion émergente, le directeur introduit verbalement la section comme si aucune faute n'avait eu lieu et donne la parole à la journaliste. Cette continuation du discours permet que la faute ne soit pas relevée ou passe inaperçue pour les auditeurs $[20]^{\dagger \dagger}$. Cela se manifeste avec des énoncés qui pourraient être considérés comme tout à fait normaux, d'autant plus qu'ils incluent une structure syntaxique routinière : «c'est le premier secret de darcy quinn à caracol». Quant au plan phonétique, un certain dérangement pourrait être entendu mais il faudrait prêter une attention accrue pour l'identifier dans un format exclusivement sonore, comme celui dans lequel l'auditoire le reçoit. En effet, le moment où DAR lève le bras en faisant un mouvement de reproche à l'opérateur technique (fig. 6) coïncide avec une intonation accrue sur qUINn (1.5), mais pour les auditeurs cela pourrait correspondre juste à une emphase sur le nom de la journaliste, car n'ayant pas accès au visuel ils ne peuvent pas voir le mouvement corporel. De cette manière, le directeur de l'émission vise à garder l'ethos de professionnel médiatique vis-à-vis de l'auditoire. L'image projetée garantie la responsabilité et la professionnalité en lui accordant de la crédibilité, facteur qui à long terme permet la fidélisation du public et donc l'existence du programme en lui-même. Pendant le passage à l'antenne de la journaliste qui développe cette section le directeur continue à faire des gestes qui exposent son ressenti à l'opérateur technique.

(10) Prise de parole de DCY
31. DCY regardez darío dans les prochains jours francisco
mire darío en los próximos días francisco
32. DCY ricaurte: (.) sera ci*tado po:r (.) la ricaurte: (.) sera convoqué pa:r (.) la
da
* lève son regard vers
l'opérateur, lève les avant-bras tout en joignant les doigts de deux mains et les agitant ${ }^{\neq \neq}--->$

\section{DCY}
declaración por\# la: fiscalía (.) y se
fig

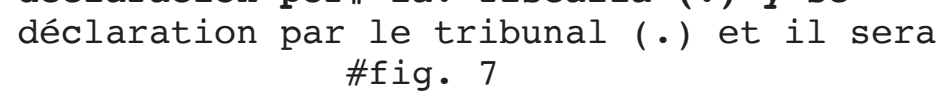

\footnotetext{
${ }^{+\dagger}$ Goffman a étudié la manière dont les journalistes et animateurs de radio corrigeaient leurs fautes. Cette réparation était faite parfois avec humour, parfois avec une mise en évidence de l'erreur, parfois avec l'exposition des onomatopées et sons propres à l'hésitation et même parfois avec une continuation du discours ainsi la faute n'est pas relevée ou passe inaperçue.

‡¥ Ce geste a pour signification culturelle une insulte : «boludo», qui pourrait être traduit par « stupide », est en effet un mot avec une connotation péjorative.
} 
34. DCY decidira* si hay o no

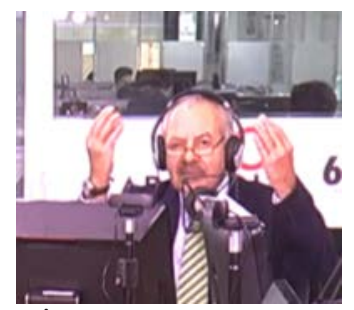

Fig. 7

da

decidé s'il y a ou pas

De cette manière, DAR réalise alors un « shift of footing » selon les termes de Goffman [20]. Plus précisément : " a change of footing implies a change in the alignment we take up to ourselves and others present as expressed in the way we manage the production or reception of an utterance » $[20$, p. 128]. Il est passé d'une énonciation bidirectionnelle adressée à l'audience et à DCY, afin de lui donner la parole en introduction de section, à une interaction unidirectionnelle adressée à l'opérateur technique. Il est intéressant de remarquer que cette double adresse a commencé à se réaliser en simultané pendant son passage à l'antenne, comme nous l'avons observé dans l'extrait (9). En effet, dans cet extrait il s'adresse en lignes 4 et 5 verbalement à l'antenne, donc aux écouteurs et à DCY, et corporellement à l'opérateur technique. Cela met en évidence la présence des différents cadres de participation au sein d'une émission radiophonique. D'un côté, ce qui est audible concerne l'équipe journalistique et les auditeurs. C'est donc dans ce contexte que l'image que l'entité de production souhaite construire se matérialise, faisant appel aux éléments du dispositif d'énonciation [2]. De l'autre côté, ce qui est visible implique le personnel en studio de diffusion mais aussi celui en studio de production, avec lequel différents canaux de communication sont ouverts. Ainsi, l'émergence de communications parallèles cachées au grand public est favorisée.

Après la fermeture des microphones, DAR adresse une réclamation verbale à l'opérateur technique accompagnée de gestes et de mouvements de la tête.

\section{(11) Réclamation verbale}

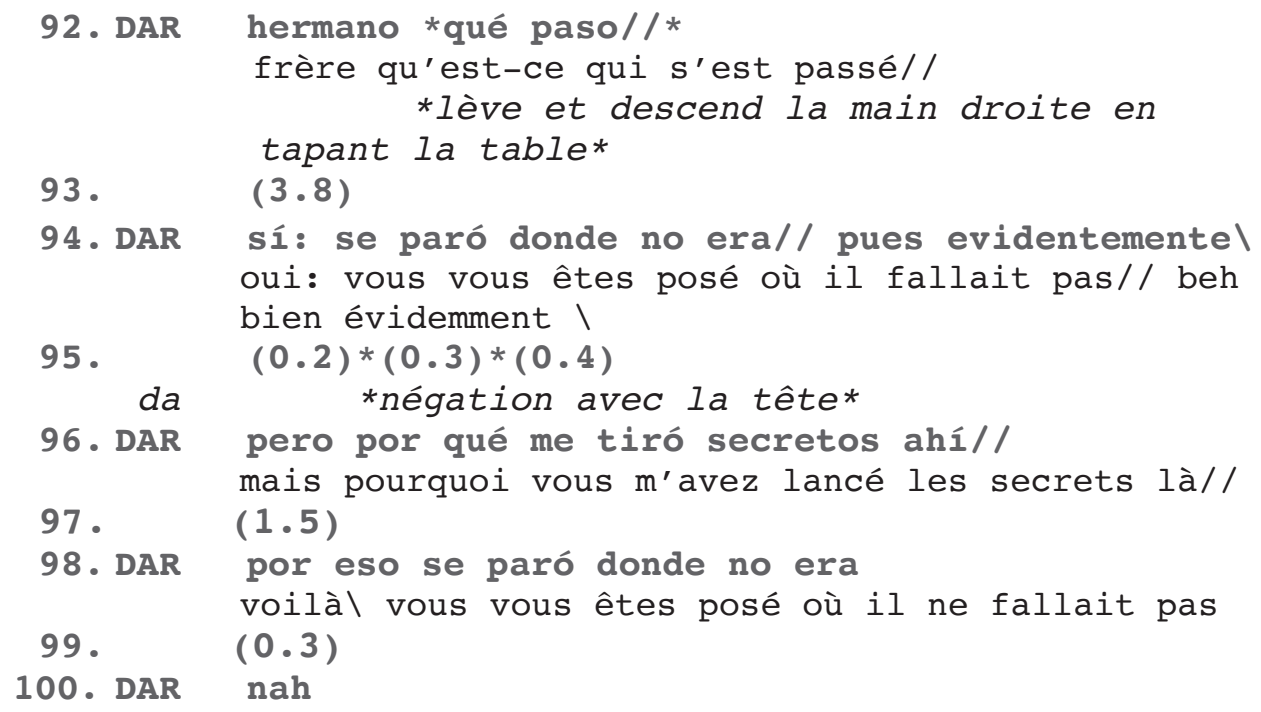

94. DAR sí: se paró donde no era// pues evidentementel oui: vous vous êtes posé où il fallait pas// beh bien évidemment ।

95. $(0.2) *(0.3) *(0.4)$

da *négation avec la tête*

96. DAR pero por qué me tiró secretos ahí//

mais pourquoi vous $\mathrm{m}^{\prime}$ avez lancé les secrets là//
98. DAR por eso se paró donde no era
voilàl vous vous êtes posé où il ne fallait pas

99. (0.3)

100. DAR nah 
Même si nous n'avons pas accès aux tours de parole de l'opérateur technique, car ils sont médiés par le casque, nous pouvons comprendre par les énoncés de DAR qu'il a essayé de justifier sa faute mais que cela n'a pas convaincu le directeur. Face à l'erreur, il a été contraint à continuer l'émission et donc à donner suite à la section annoncée par le jingle diffusé par

Ainsi, cet extrait donne à voir à quel point il est important de préserver la face [21] à la radio, et cela ne se réduit pas à un point de vue exclusivement individuel, même si la conservation de la professionnalité entre en jeu, il y a aussi une double identité qui se mobilise. De fait, chaque journaliste ou professionnel médiatique représente la station entière.

\section{Conclusion}

Dans les deux cas présentés, il y a un contraste entre une « communication émotive », où apparaît une structure intentionnellement organisée, et une émotion vécue qui provoquerait normalement une « communication émotionnelle » pour reprendre la distinction de Plantin [1] mais qui là, au contraire, a été cachée à l'auditoire, destinataire ultime de l'interaction médiatique. En revanche, elle a été affichée à l'opérateur technique puisque son action était la source de l'émotion.

Pour le premier cas, nous avons observé un exemple de construction d'émotion chez l'auditoire. Pour cela, des procédures au plan discursif, prosodique et gestuel ont été mobilisées. Elles font partie des stratégies de «captation», en termes de Charaudeau «destinées à déclencher des imaginaires émotionnels» [22] en poursuivant la visée pathémique intentionnellement déclenchée.

Pour le deuxième cas, contrario, l'émotion ressentie n'est pas intentionnellement affichée à l'auditoire. L'objectif est de ne pas « perdre la face » d'après Goffman [21] vis-à-vis du public, car cela implique des enjeux professionnels débouchant sur la crédibilité et le savoirfaire professionnel des journalistes et par ricochet de la chaîne entière.

Ainsi, à travers l'étude séquentielle de ces deux cas, l'objectif a été d'observer comment l'étude de l'intentionnalité émotionnelle permet de mettre à jour certains procédés qu'on peut envisager comme propres à la professionnalité journalistique comme la construction en permanence de l'ethos d'expert mais aussi l'élaboration du pathos selon la visée recherchée.

Enfin, il convient de remarquer que l'accès aux données visuelles dans ce type de recherche permet de connaître les multiples procédures mobilisées dans le processus de construction de la parole radiophonique. Cela enrichit l'analyse car, comme nous l'avons observé dans les cas étudiés, l'aspect corporel, même s'il peut être accompagné par des changements prosodiques, ne peut pas être intégralement interprété sans avoir accès au format visuel.

\section{Bibliographie}

1. C. Plantin, C. Les bonnes raisons des émotions. Principes et méthode pour l'étude du discours émotionné. (Peter Lang, Berne, 2011)

2. L. Fauré et N. Smati. Le discours. Dans F. Antoine (dir.), Analyser la radio. Méthodes et mises en pratique. 99-104 (De Boeck Supérieur, Bruxelles, 2016)

3. L. Quéré. Faut-il abandonner l'étude de la réception ? Point de vue. Dans : Réseaux, 14, 79, 31-37 (Recherches anglaises, 1996)

4. P. Charaudeau, Pathos et discours politique. Dans M. Rinn, (Ed.), Émotions et discours : L'usage des passions dans la langue, 49-58. (Presses universitaires de Rennes, Rennes, 2008) 
5. P. Charaudeau, La pathémisation à la télévision comme stratégie d'authenticité. Dans $\mathrm{C}$. Plantin, M. Doury, et V. Traverso (dirs.), Les émotions dans les interactions. (Presses universitaires de Lyon, Lyon, 2000)

6. P. Scannell. (Éd.). The relevance of talk. Dans Broadcast talk, 1-13 (Sage Publications, 1991)

7. I. Hutchby. Media talk: Conversation analysis and the study of broadcasting. (Open University Press, 2006)

8. M. Burger et A. Auchlin. Quand le parler radio dérange : remarques sur le phono-style de France Info. Dans M. Broth, M. Frosgren, C. Norén et F. Sullet-Nylander (sld.), Le Français parlé des médias. Actes du colloque de Stockholm 8-12 juin 2005, 97-111. (Acta Universitatis Stockholmiensis, Stockhom, 2007)

9. H. Glevarec. France Culture à l'oeuvre: Dynamique des professions et mise en forme radiophonique. (CNRS, 2001)

10.K. Lan Hing Ting et D. Voilmy L'évitement du blanc radiophonique comme accomplissement multimodal. Communication. Information médias théories pratiques, Vol.27/2, 166-184. (2010).

11. L. Mondada et F. Oloff, Passer à l'antenne : arrangements technologiques et interactionnels préparant la prise de parole en direct Cahiers de praxématique, 61 (2013).

12. H. Garfinkel. Studies in ethnomethodology. (Prentice-Hall, Inc., 1967)

13. A. Duranti. Linguistic anthropology. (Cambridge University Press, New York, 1997)

14. D. Maingueneau, Discours et analyse du discours : Une introduction. (Armand Collin, Paris, 2014)

15. L. Mondada, Multiple Temporalities of Language and Body in Interaction: Challenges for Transcribing Multimodality. Research on Language and Social Interaction, 51, 1, 85-106 (2018)

16. P. Drew, Turn Design. Dans J. Sidnell \& T. Stivers (éds), The Handbook of conversation analysis, 131-149 (Blackwell Publishing, Oxford, 2013).

17. C. Plantin, V. Traverso et L. Vosghanian. Parcours des émotions en interaction. In M. Rinn (dir.), Émotions et Discours. L'usage des passions dans la langue, 141-162. (Presses universitaires de Rennes, 2008)

18. L. Quéré. Des miroirs équivoques: Aux origines de la communication moderne (Montaigne, Aubier, 1982)

19. L. Mondada. Transcribing silent actions: A multimodal approach of sequence organization. Social Interaction. Video-Based Studies of Human Sociality, 2, 1 (2019)

20. E. Goffman Forms of Talk. (University of Pennsylvania Press, Pennsylvania, 1981)

21. E. Goffman La mise en scène de la vie quotidienne 1. La présentation de soi (Ed. de Minuit, Paris, 1973)

22. P. Charaudeau, Ce que communiquer veut dire. Revue des Sciences humaines, 51 (1995). 